\title{
CARDIOLOGÍA DE LA MUJER
}

\section{Hipertensión arterial y embarazo}

\author{
High blood pressure and pregnancy
}

\section{Ana G. Múnera-Echeverri1 ${ }^{1 *}$, Edison Muñoz-Ortiz³ y Jaime A. Ibarra-Burgos ${ }^{4}$}

${ }^{1}$ Servicio Cardiología no Invasiva, Hospital General de Medellín; ${ }^{2}$ Grupo de Investigación, Hospital General de Medellín, Universidad CES; ${ }^{3}$ Clínica Cardioobstétrica, Unidad Funcional Cardiopulmonar y Vascular Periférico, Hospital Universitario San Vicente Fundación, Universidad de Antioquia; ${ }^{4}$ Servicio de Medicina Interna, Universidad CES. Medellín, Colombia

\section{Resumen}

Los trastornos hipertensivos asociados al embarazo son una complicación frecuente y causa importante de morbimortalidad materno-fetal. Realizar un diagnóstico oportuno, definir la necesidad de inicio de terapias farmacológicas orales o intravenosas, así como las indicaciones de cuidado en centros de referencia, es el papel fundamental de los diferentes médicos que pueden verse enfrentados a este tipo de pacientes. Se propone una revisión completa de la clasificación, el enfoque diagnóstico y los aspectos fundamentales del tratamiento durante el embarazo, el parto y el posparto de los trastornos hipertensivos asociados al embarazo, así como las consecuencias futuras en el riesgo cardiovascular de la mujer con este tipo de enfermedades.

Palabras clave: Hipertensión arterial. Embarazo. Preeclampsia.

\section{Abstract}

Hypertensive disorders associated with pregnancy are a frequent complication and an actual cause of maternal-fetal morbidity and mortality. Perform a timely diagnosis, defining the need to start oral or intravenous pharmacological therapies and the indications for care in referral centers is the different physicians' fundamental role. They can meet with this type of patient. A complete review of the classification, the diagnostic approach, and the fundamental aspects of the treatment during pregnancy, childbirth, and postpartum of the hypertensive disorders associated with pregnancy is reviewed and the future consequences in the woman's cardiovascular risk.

Key words: Arterial hypertension. Pregnancy. Preeclampsia.

\section{Introducción}

Los trastornos hipertensivos asociados al embarazo complican el 5 al $10 \%$ de las gestaciones en el mundo y son la principal causa de morbimortalidad materna, fetal y neonatal. Los riesgos maternos incluyen abrupcio placentario, enfermedad cerebrovascular, falla multiorgánica y coagulación intravascular diseminada. La preeclampsia genera en el feto aumento del riesgo de retardo del crecimiento intrauterino (25\%), prematuridad $(27 \%)$ y muerte intrauterina $(4 \%)^{1}$. Es posible que la frecuencia de trastornos hipertensivos asociados al embarazo sea mayor y se subestime actualmente, debido al incremento en la frecuencia de obesidad y

\section{Correspondencia:}

*Ana G. Múnera-Echeverri

E-mail: anagm@une.net.co
Fecha de recepción: 19-05-2020

Fecha de aceptación: 14-08-2020

DOI: 10.24875/RCCAR.M21000002
Disponible en internet: 19-03-2021 Rev Colomb Cardiol. 2021;28(1):3-13 www.rccardiologia.com 0120-5633 / C 2020 Sociedad Colombiana de Cardiología y Cirugía Cardiovascular. Publicado por Permanyer. Este es un artículo open access bajo la licencia CC BY-NC-ND (http://creativecommons.org/licenses/by-nc-nd/4.0/). 
diabetes en mujeres en edad reproductiva, así como la edad materna avanzada al momento de embarazarse $^{1,2}$. En Colombia, según publicaciones del 2013, se estimaba que $35 \%$ de las muertes maternas se asociaban a trastornos hipertensivos asociados al embarazo ${ }^{3}$.

La preeclampsia es una enfermedad multisistémica, multifactorial, dinámica y progresiva, que puede presentarse durante la gestación o el posparto. El manejo apropiado requiere la evaluación frecuente de las manifestaciones de gravedad. Adicionalmente, los trastornos hipertensivos asociados al embarazo se consideran como factores de riesgo cardiovascular específicos de la mujer, ya que se asocian con riesgo de enfermedad cardiovascular en el futuro $0^{1,4,5}$.

\section{Fuente y criterios de selección}

Se realizó una búsqueda de la literatura científica sobre los trastornos hipertensivos asociados al embarazo, en las bases de datos de Medline (A través de PubMed), Librería de Cochrane, Scopus, Science Direct y SciELO. En la estrategia de búsqueda se incluyeron los términos MeSH "Pre-Eclampsia" OR "Hypertension, Pregnancy-Induced" OR "Eclampsia" OR "HELLP syndrome". La búsqueda se limitó por tiempo a aquellos artículos publicados a partir del año 2010 y solo se tuvieron en cuenta aquellos publicados en inglés o español. La búsqueda fue ampliada a partir de las listas bibliográficas de los trabajos encontrados. Así mismo, referencias clave adicionales fueron recuperadas a partir de la base de datos personal de cada uno de los coautores. Finalmente, para esta revisión se extrajo información a partir de 12 guías de práctica clínica, 3 consensos de expertos, 2 revisiones sistemáticas de la literatura y meta-análisis, 3 ensayos clínicos aleatorizados, 2 estudios de cohortes y 4 revisiones narrativas.

\section{Definiciones y clasificación}

Pese a las diferencias en definiciones de hipertensión arterial (HTA) en adultos entre las sociedades europea ${ }^{5}$ americana ${ }^{6}$, la definición de HTA en la gestante es relativamente unánime entre la Sociedad Europea de Cardiología (ESC) y el Colegio Americano de Ginecoobstetricia (ACOG), y se considera cuando la cifra de PA sistólica (PAS) es mayor o igual a $140 \mathrm{~mm}$ $\mathrm{Hg}$ y la PA diastólica (PAD) mayor o igual a $90 \mathrm{~mm} \mathrm{Hg}{ }^{1,5,7}$. A su vez, los trastornos hipertensivos asociados al embarazo, según las cifras tensionales, se clasifican como leves cuando la PAS está entre 140-149 mm Hg y la PAD entre 90-109 mm Hg, y graves si la PAS es $\geq 160 \mathrm{~mm} \mathrm{Hg}$ y o la PAD $\geq 110 \mathrm{~mm} \mathrm{Hg}^{1,5,7}$.

Existen varias clasificaciones de acuerdo con las diferentes sociedades que desarrollan guías sobre el tema, pero se propone seguir la recomendada por las guías de enfermedad cardiaca y embarazo de la ESC ${ }^{1}$, dado que es muy similar a la propuesta de la $\mathrm{ACOG}^{7,8}$ y de la Sociedad Canadiense de Cardiología ${ }^{9}$. Según esta propuesta, los trastornos hipertensivos asociados al embarazo se clasifican en cuatro grupos: HTA crónica, hipertensión gestacional, preeclampsia y preeclampsia asociada a HTA crónica ${ }^{1,7-9}$. Debe tenerse presente que el esfigmomanómetro de mercurio es considerado el estándar de la medición indirecta no invasiva de la presión y ha sido validado contra la medición intraarterial de la presión; sin embargo, tiene la desventaja que el mercurio es neurotóxico y contaminante ambiental. En cuanto a los esfigmomanómetros oscilométricos un número limitado ha sido validado para uso en embarazadas y la mayoría han demostrado ser imprecisos en preeclampsia.

\section{Hipertensión antes del embarazo (HTAC crónica)}

Es aquella que precede el embarazo o aparece antes de las 20 semanas de gestación. En estas pacientes debe descartarse HTA secundaria antes de considerar HTA esencial. Idealmente, deben recibir asesoramiento antes del embarazo, manejo con fármacos apropiados y ser referidas a centros obstétricos para manejo de la gestación por riesgo de desarrollar preeclampsia.

\section{Hipertensión gestacional}

Hipertensión detectada luego de la semana 20 de gestación en ausencia de manifestaciones de preeclampsia. Es necesario evaluar proteinuria, descartar por laboratorio disfunción de órganos y evaluar por ultrasonido el crecimiento fetal en todas las mujeres asintomáticas con inicio de HTA luego de las 20 semanas de gestación. Existe riesgo de progresión a preeclampsia en cerca del $25 \%$ de las pacientes.

\section{Preeclampsia}

Inicio de HTA luego de la semana 20 de gestación con proteinuria. La presentación puede ser incluso en el postparto. En ausencia de proteinuria la 
Tabla 1. Criterios diagnósticos de preeclampsia

\begin{tabular}{|c|c|}
\hline Hipertensión arterial & $\begin{array}{l}\text { - La HTA se define como PAS }>140 \mathrm{~mm} \mathrm{Hg} \text { o PAD }>90 \mathrm{~mm} \mathrm{Hg} \text { en dos mediciones separadas } \\
\text { por un período de reposo de al menos } 15 \text { minutos. } \\
\text { - HTA grave: PA > } 160 / 110 \mathrm{~mm} \mathrm{Hg} \text {. } \\
\text { - La PA debe ser medida en reposo, con el brazo a nivel del corazón. Si la madre está en } \\
\text { supino, debe ser en posición lateral izquierda. }\end{array}$ \\
\hline Proteinuria & $\begin{array}{l}\text { - } 300 \mathrm{mg} \text { en orina de } 24 \text { horas. } \\
\text { - Relación proteínas/creatinina en una muestra de orina }>30 \mathrm{mg} / \mathrm{mmol} 0>0.3 \mathrm{mg} / \mathrm{dl} \text { indica } \\
\text { proteinuria significativa. } \\
\text { - Si no hay disponibilidad de estudio en orina de } 24 \text { horas o relación proteínas/creatinina, es } \\
\text { razonable iniciar manejo cuando se presenta (proteínas }>1+\text { ) y existe sospecha clínica alta. } \\
\text { No obstante, debe tenerse en cuenta la baja sensibilidad de esta prueba. }\end{array}$ \\
\hline \multicolumn{2}{|c|}{ En ausencia de proteinuria, el inicio de hipertensión y uno de los siguientes } \\
\hline Trombocitopenia & $<100.000 /$ microlitro \\
\hline Insuficiencia renal & $\begin{array}{l}\text { Creatinina }>1.1 \mathrm{mg} / \mathrm{dl} \text { o dos veces por encima del valor de referencia en ausencia de } \\
\text { enfermedad renal. }\end{array}$ \\
\hline Compromiso de la función hepática & $\begin{array}{l}\text { Transaminasas al doble de lo normal. } \\
\text { Dolor en epigastrio o cuadrante superior derecho en ausencia de diagnóstico alternativo }\end{array}$ \\
\hline \multicolumn{2}{|l|}{ Edema pulmonar } \\
\hline Síntomas visuales o neurológicos & Cefalea que no mejora con medicamentos en ausencia de diagnóstico alternativo \\
\hline
\end{tabular}

preeclampsia se diagnostica por la presencia de HTA asociada a daño de órgano blanco (tabla 1). La enfermedad grave puede desarrollarse rápidamente aún en mujeres con manifestaciones iniciales leves. Debe tenerse en cuenta el empeoramiento de síntomas visuales , dolor abdominal, náuseas, vómito y cefalea. En la tabla 2 se resumen las manifestaciones clínicas y de laboratorio de la preeclampsia grave. Se considera marcador de gravedad el inicio temprano $<34$ semanas.

\section{Preeclampsia asociada a hipertensión arterial crónica}

Se presenta en mujeres que tienen HTA conocida, pero desarrollan empeoramiento de la HTA en combinación con proteinuria, nueva disfunción de órgano o disfunción útero-placentaria.

En el espectro de la preeclampsia se encuentran dos alteraciones adicionales que corresponden a las formas de presentación más grave de los trastornos hipertensivos asociados al embarazo, los cuales son ${ }^{1,7-9}$ el síndrome HELLP, que escribe una serie de manifestaciones, entre ellas, hemólisis (anemia con evidencia de hemólisis), elevación de las enzimas hepáticas (transaminasas mayores a dos veces el rango normal) y trombocitopenia, y la eclampsia, que se refiere a la
Tabla 2. Manifestaciones de la preeclampsia grave

Presión arterial > 160/110 mm Hg

Trombocitopenia (plaquetas $<100.000 /$ microlitro )

Compromiso de la función hepática: enzimas hepáticas elevadas dos veces el valor normal; dolor persistente severo en el cuadrante superior derecho o epigástrico.

Insuficiencia renal progresiva: creatinina $>1.1 \mathrm{mg} / \mathrm{dl}$ o dos veces por encima del valor de referencia en ausencia de enfermedad renal.

Edema pulmonar

Inicio de síntomas visuales o neurológicos.

presencia de convulsiones tónico-clónicas focales o multifocales en la mujer embarazada, durante el parto o el postparto sin otra causa atribuible, como epilepsia, isquemia cerebral, hemorragia intracraneana o uso de medicamentos. La búsqueda etiológica de las convulsiones no debe retardar el inicio del tratamiento para la eclampsia y todas las convulsiones generalizadas luego de la semana 20 de gestación deben ser manejadas como eclampsia, excepto que exista una causa etiológica diferente. Los síntomas más comunes que preceden la eclampsia son cefalea grave, trastornos visuales y náuseas en el 7 al $83 \%$ de las 
pacientes. Se ha descrito que hasta la tercera parte de las pacientes con eclampsia no estaban hipertensas antes de la convulsión.

\section{Fisiopatología}

La fisiopatología de los trastornos hipertensivos asociados al embarazo no está totalmente explicada. En el embarazo, el sistema renina-angiotensina está regulado y la resistencia vascular sistémica disminuye. Como resultado, la presión arterial (PA) inicialmente disminuye comenzando desde la semana 7 de gestación. La PA materna comienza a incrementar luego en el tercer trimestre, llegando a niveles cercanos a los previos al embarazo $0^{1,10}$.

El evento desencadenante para desarrollar eventos hipertensivos en el embarazo es la invasión anormal del citotrofoblasto en las arterias espirales, lo cual lleva a reducción de la perfusión útero-placentaria. La isquemia placentaria resultante origina una cascada de eventos inflamatorios, disrupción del balance entre factores angiogénicos y agregación plaquetaria, lo cual finalmente conduce a disfunción endotelial que clínicamente se expresa con elevación de la PA ${ }^{10}$. El desbalance entre factores se ha propuesto como herramienta diagnóstica ${ }^{10,11}$ y estaría involucrado en la reducción de la síntesis de óxido nítrico ${ }^{10}$.

La preeclampsia se asocia con incremento de la resistencia vascular sistémica, incremento de la postcarga ventricular izquierda y presiones ventriculares con aparición de disfunción diastólica, reducción del gasto cardíaco, strain anormal y dilatación auricular izquier$\mathrm{da}^{12}$. Por otro lado, como el flujo sanguíneo disminuye, se reduce la filtración glomerular. La causa exacta de las convulsiones no está bien explicada, pero se han propuesto la encefalopatía hipertensiva y la isquemia secundaria. Los cambios visuales pueden ocurrir por edema secundario de los hemisferios cerebrales posteriores ${ }^{10}$.

\section{Enfoque diagnóstico}

Las mujeres con HTA crónica deberían tener una evaluación previa al embarazo para identificar posible daño de órgano blanco, definir necesidad de estudios de HTA secundaria, optimizar comorbilidades y realizar cambios terapéuticos en el estilo de vida que permitan mejorar factores de riesgo modificables. Adicionalmente, deberían tener ajustes del tratamiento antihipertensivo de manera que logren alcanzar las metas de control óptimo y suspender medicamentos deletéreos
Tabla 3. Riesgos de la hipertensión crónica en el embarazo

\begin{tabular}{|l|l|}
\hline Maternos & Fetales y neonatales \\
\hline Muerte & Mortinato o muerte perinatal \\
$\begin{array}{l}\text { Ataque cerebrovascular } \\
\text { Edema pulmonar }\end{array}$ & Restricción del crecimiento \\
Falla renal & Parto pretérmino \\
Infarto del miocardio & Anomalías congénitas \\
Preeclampsia & \\
Abrupcio de placenta & \\
Parto por cesárea & \\
Hemorragia posparto & \\
Diabetes gestacional &
\end{tabular}

Tabla 4. Estudios basales en mujer con hipertensión crónica que desea embarazarse o en primera evaluación en el embarazo

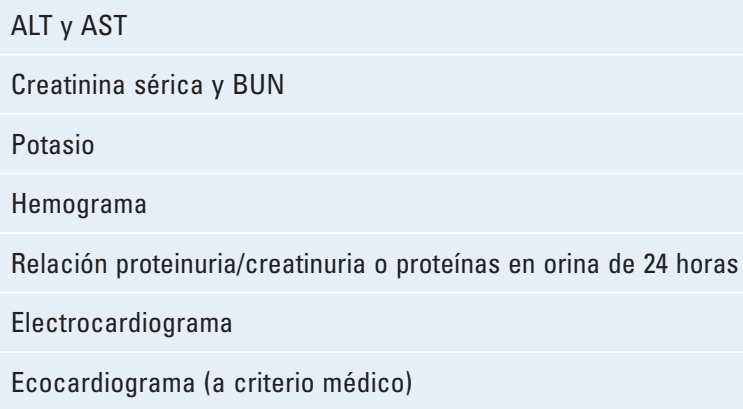

ALT: alanino aminotransferasa, AST: aspartato aminotransferasa, BUN: nitrógeno ureico en sangre.

para el embarazo, como inhibidores de la enzima convertidora de angiotensina (IECA), antagonistas del receptor de angiotensina II (ARA-II), inhibidores de la renina o antialdosterónicos. Además, en esta evaluación es fundamental explicarle a la paciente los riesgos asociados de la HTA crónica durante el embarazo $(\text { tabla } 3)^{8}$.

Adicional a una historia clínica y un examen físico completos, la evaluación en busca de daño de órgano blanco debe incluir paraclínicos basales, a realizar idealmente antes del embarazo y en el peor de los escenarios al momento de la detección del embarazo (tabla 4) $)^{7,8}$.

En las pacientes con o sin antecedente de HTA que tengan cambios en sus cifras tensionales o cuadro clínico que sugiera preeclampsia sobreagregada, se requerirán estudios para identificar los hallazgos de gravedad por compromiso de órgano blanco mencionados en la tabla 2, además de los estudios que permitan determinar el estado fetal, como la ecografía obstétrica y el estudio doppler de arterias umbilicales ${ }^{1,7,9}$. 
Tabla 5. Valores de la monitorización ambulatoria de presión arterial según la edad gestacional. Rangos de presión arterial con valor superior normal en paréntesis

\begin{tabular}{|c|c|c|c|c|}
\hline Edad gestacional (semanas) & $9-17$ & $18-22$ & $26-30$ & $31-40$ \\
\hline PAS mm Hg & $101-118(121)$ & $96-127$ (126) & $97-133(128)$ & $103-136(131)$ \\
\hline PAD mm Hg & $60-71(73)$ & $56-78(76)$ & $56-84(78)$ & $57-85(82)$ \\
\hline
\end{tabular}

PAS: presión arterial sistólica, PAD: presión arterial diastólica.

Tabla 6. Factores de riesgo de preeclampsia

\begin{tabular}{|l|l|l|}
\hline FR Alto & FR Moderado & FR Bajo \\
\hline Historia de pre-eclampsia & Nuliparidad & Parto a término previo no complicado \\
\hline Gestación múltiple & Obesidad (IMC>30 ACOG, 35 ESC) & \\
\hline Hipertensión arterial crónica & Historia familiar de preeclampsia (madre o hermana) & \\
\hline Diabetes mellitus & $\begin{array}{l}\text { Características sociodemográficas (afroamericana, bajo } \\
\text { nivel económico) }\end{array}$ & \\
\hline Enfermedad renal & Edad $\geq 35$ años (ACOG) - 40 años (ESC) & \\
\hline Enfermedad autoinmune (SAF - LES) & $\begin{array}{l}\text { Factores de historia personal (bajo peso al nacer o } \\
\text { pequeño para edad gestacional, resultado adverso en } \\
\text { embarazo previo, intervalo entre embarazo }>10 \text { años) }\end{array}$ & \\
\hline
\end{tabular}

ACOG: Colegio Americano de Ginecoobstetricia; ESC: Sociedad Europea de Cardiología; FR: Factores de riesgo; IMC: índice de masa corporal; LES: lupus eritematoso sistémico; SAF: síndrome antifosfolípido.

La HTA de bata blanca puede presentarse en el embarazo y no es una condición totalmente benigna; la mitad de estas pacientes pueden desarrollar HTA gestacional y el $8 \%$ preeclampsia. En estos casos está indicado el monitoreo ambulatorio de la presión arterial (MAPA), el cual también es útil para el diagnóstico de HTA nocturna, esta última asociada con mayor riesgo de complicaciones maternas y fetales. Otras indicaciones de MAPA durante el embarazo son variación significativa entre las tomas de PA, detección de hipertensión arterial enmascarada, hipertensión preexistente o crónica, antecedentes de preeclampsia, diabetes y PA en el límite superior normal. El protocolo empleado para la realización de MAPA durante el embarazo es similar en las no embarazadas. Los valores de PA según la edad gestacional se presentan en la tabla $5^{13,14}$.

Estudios para tratar de predecir preeclampsia, como el doppler de arterias uterinas a las 20 a 22 semanas, o la relación sFlt-1:PIGF, tienen recomendaciones discordantes, de modo que la ESC los sugiere ${ }^{1}$, mientras la ACOG y las guías colombianas no los recomiendan ${ }^{3,7}$.

\section{Prevención de preeclampsia}

En los últimos treinta años se han estudiado diferentes estrategias para tratar de prevenir los trastornos hipertensivos asociados al embarazo, con base en terapias nutricionales, suplementos vitamínicos y fármacos.

El ácido acetilsalicílico en dosis de 81 a $150 \mathrm{mg}$ iniciando entre la semana 11-14 y manteniendo hasta la semana 36-37, se recomienda en pacientes con un factor de riesgo alto de preeclampsia o dos factores de riesgo moderado (tabla 6), ${ }^{1,115}$.

La administración de calcio (1.5-2.0 g/día) puede ser útil para prevenir la preeclampsia de acuerdo con el resultado de un meta-análisis en población con ingesta baja de calcio ${ }^{16}$. Los antioxidantes como vitamina $C$ y E no son efectivos, así como tampoco hay beneficio con dieta baja en sal, reposo en cama o metformina.

\section{Tratamiento}

El tratamiento busca mejorar los desenlaces materno-fetales, con énfasis en el impacto en la mortalidad 
materna asociada a estas enfermedades, pero este dependerá del momento del embarazo en el que se pretenda intervenir ${ }^{8}$.

\section{Manejo preconcepcional y al inicio del embarazo}

Como se mencionó previamente, lo ideal es que toda mujer antes de embarazarse tenga una evaluación preconcepcional para identificar los factores de riesgo para trastornos hipertensivos asociados al embarazo. En pacientes con HTA conocida es más relevante aún dicha consulta preconcepcional con el fin de optimizar el control de la presión arterial, suspender los antihipertensivos con potencial teratogénico y realizar estudios basales que permitan conocer el estado de salud de la paciente (tabla 4). Adicionalmente, se debe insistir a todas las pacientes en cambios terapéuticos en el estilo de vida, no solo para un embarazo sin complicaciones, sino, en general, para una adecuada salud cardiovascular ${ }^{1,7,8}$.

\section{Tratamiento de la hipertensión durante el embarazo}

Una vez se diagnostica un trastorno hipertensivo asociados al embarazo, el manejo está determinado por la forma de presentación y por las manifestaciones de gravedad $^{7}$. El tratamiento busca un balance entre los riesgos maternos de continuar la gestación, los riesgos de la intervención materna y los riesgos fetales de la prematuridad. Si la decisión es continuar la gestación, los objetivos del tratamiento son mantener una presión arterial en rangos seguros y monitorizar de manera estrecha los signos de deterioro materno-fetal ${ }^{1,9,10}$.

Se recomienda hospitalizar a las pacientes con diagnóstico de HTA gestacional o preeclampsia para completar la evaluación materno-fetal, estabilizar la presión arterial y organizar un plan de trabajo y seguimiento ${ }^{1-3,7}$. Así mismo, debe evaluarse el riesgo de trombosis venosa profunda, teniendo presente que la mayoría debería recibir tromboprofilaxis ${ }^{1}$.

\section{Hipertensión no grave en el embarazo}

Cuando la presión arterial es mayor o igual a $140 / 90 \mathrm{~mm} \mathrm{Hg}$, pero menor a 160/110 mm Hg en una gestante, se habla de HTA leve (no grave). En este grupo de pacientes, se recomienda hospitalizar para hacer el enfoque diagnóstico y estudios que permitan establecer con certeza la gravedad. Usualmente, tienen un tratamiento más conservador, siempre y cuando no exista compromiso de órgano blanco, pues de lo contrario, este sería un criterio de gravedad más allá del valor de presión arterial $\left.\right|^{1,7-9}$. Existen algunas discrepancias entre diferentes sociedades sobre el valor para definir el inicio del tratamiento farmacológico; por ejemplo, las guías colombianas lo recomiendan con presión arterial $\geq 150 / 100 \mathrm{~mm} \mathrm{Hg}^{3}$, ACOG si la presión arterial está elevada en forma persistente $\geq 150 / 110 \mathrm{~mm} \mathrm{Hg}$ en presencia de comorbilidades ${ }^{7}$, mientras que las guías ESC consideran la terapia farmacológica en toda gestante con HTA crónica y presión arterial $\geq 150 / 95 \mathrm{~mm} \mathrm{Hg}$, con la aclaración que la terapia farmacológica se debe iniciar en valores de presión arterial $\geq 140 / 90 \mathrm{~mm} \mathrm{Hg}$ en pacientes con hipertensión gestacional (con o sin proteinuria), HTA crónica con preeclampsia superpuesta o en presencia de daño de órgano blanco subclínico o síntomas en cualquier momento del embarazo ${ }^{1}$. Por su parte, el Instituto Nacional de Salud y excelencia en el cuidado del Reino Unido (NICE), considera tratamiento farmacológico en todas las pacientes con presión arterial $\geq 140 / 90 \mathrm{~mm} \mathrm{Hg}{ }^{17}$. Un meta-análisis que trató de definir la indicación de tratamiento antihipertensivo en paciente con HTA no grave en el embarazo, comparó la administración de tratamiento antihipertensivo contra placebo, sin encontrar diferencias en la aparición de preeclampsia, muerte fetal, restricción de crecimiento intrauterino ni parto pretérmino, pero halló menos tasas de desarrollo de HTA grave en forma significativa ${ }^{18}$. Así mismo, un estudio en gestantes con hipertensión arterial crónica o gestacional no grave, comparó un control de cifras tensionales más estricto ( $P A D<85 \mathrm{~mm} \mathrm{Hg}$ ) versus control flexible (PAD $<100 \mathrm{~mm} \mathrm{Hg}$ ), y encontró que no había diferencias en el riesgo de pérdida de la gestación, necesidad de cuidado neonatal de alta dependencia o complicaciones maternas; sin embargo, en los desenlaces secundarios del estudio, se observó que las pacientes con una meta de control más estricto tenían menor tasa de HTA grave ${ }^{19}$. Adicionalmente, un subanálisis del estudio reportó que las pacientes que presentaron HTA grave en el seguimiento, tuvieron mayores tasas de pérdidas del embarazo, peso bajo para la edad gestacional, preeclampsia, parto pretérmino, plaquetas $<100.000 /$ microlitro y elevación de transaminasas, sin aumento de la mortalidad materna ${ }^{20}$.

Se recomienda, por tanto, monitorización estrecha en las pacientes con HTA gestacional o preeclampsia sin manifestaciones graves, con evaluación diaria de 
síntomas y movimientos fetales, mediciones seriadas de la presión arterial (mínimo 6 veces/día), recuento plaquetario, función renal y pruebas hepáticas cada 2 días. Adicionalmente, se debe medir la proteinuria una vez a la semana con el fin de detectar lo más temprano posible la presencia de criterios de gravedad que indican el inicio de tratamiento farmacológico si la paciente no lo venía recibiendo ${ }^{3}$. Se debe entender que las clasificaciones son, en general, ilustrativas, dado que la enfermedad es dinámica, y una forma leve puede evolucionar a una grave.

\section{Tratamiento antihipertensivo en hipertensión arterial no grave}

Como se había mencionado previamente, durante la gestación no se recomienda el uso de IECA, ARA-II, inhibidores de la renina 0 antialdosterónicos ${ }^{1,8}$.

Las mujeres con HTA que están embarazadas o que planean quedar embarazadas, deben cambiar la medicación por labetalol, nifedipino o alfametildopa como terapias de primera línea, y tener opciones adicionales en caso de control inadecuado (tabla 7); estos medicamentos también son las opciones farmacológicas en HTA leve durante la gestación ${ }^{9}$. Existe evidencia que los betabloqueadores son menos efectivos para controlar la HTA en pacientes afrodescendientes, en quienes se recomienda nifedipino $0^{5,7-9}$.

En cuanto a cuál de los medicamentos de primera línea escoger, un metaanálisis de Cochrane comparó terapias antihipertensivas en gestantes con HTA no grave. Al evaluar la progresión a HTA grave, comparando metildopa contra un antihipertensivo alternativo, se encontró mejor resultado con el régimen alternativo. Al revisar la aparición de proteinuria y preeclampsia, comparando betabloqueadores y calcioantagonistas en conjunto contra metildopa, se encontraron resultados superiores con la terapia con betabloqueadores y calcioantagonistas $^{18}$. Con estos resultados, de los medicamentos de primera línea (tabla 7), los betabloqueadores y calcioantagonistas se prefieren por encima de la metildopa ${ }^{1,7-9,17}$.

Las metas de presión arterial no están bien establecidas y las recomendaciones de las diferentes sociedades son muy variables. Así, la ACOG recomienda presión arterial mayor a 120/80 mm Hg y menor de 160/110 mm Hg${ }^{7}$, NICE recomienda presión arterial $\leq 135 / 85 \mathrm{~mm} \mathrm{Hg}^{17}$, las guías canadienses PAD $<85$ $\mathrm{mm} \mathrm{Hg}$, las guías ESC no dan una recomendación ${ }^{1} y$ las guías colombianas una meta de presión arterial $<140 / 90 \mathrm{~mm} \mathrm{Hg}^{3}$.
Tabla 7. Medicamentos antihipertensivos durante el embarazo

\begin{tabular}{|l|l|l|}
\hline Primera línea & Segunda línea & Medicamentos a evitar \\
\hline Labetalol & Clonidina & IECA \\
\hline Metildopa & Hidralazina & ARA-II \\
\hline $\begin{array}{l}\text { Nifedipino de larga } \\
\text { acción }\end{array}$ & Tiazidas & Antialdosterónicos \\
\hline $\begin{array}{l}\text { Otros } \\
\text { betabloqueadores } \\
\text { (evitar atenolol) }\end{array}$ & & \\
\hline
\end{tabular}

ARA-II: antagonistas del receptor de angiotensina II; IECA: inhibidores de la enzima convertidora de angiotensina.

\section{Tratamiento en hipertensión arterial grave}

En presencia de HTA grave se requiere, sin duda, el inicio de terapia antihipertensiva, la cual en ausencia de signos que sugieran inminencia de eclampsia, puede iniciarse vía oral ${ }^{1,7}$. Estas pacientes requieren evaluación completa para detectar aparición de preeclampsia, tal como se mencionó en la sección de enfoque diagnóstico ${ }^{7,8}$.

La presencia de PAS > $160 \mathrm{~mm} \mathrm{Hg}$ (según la sociedad científica) o PAD >110 mm Hg es una emergencia médica, y debe ser controlada rápidamente con antihipertensivos intravenosos y dosis de mantenimiento ${ }^{7}$. El objetivo, dado el riesgo de daño de órgano blanco, es mantener la presión arterial < 150/100 mm Hg, pero con cuidado de no generar hipotensión y comprometer la circulación uteroplacentaria7. Las estrategias farmacológicas para el manejo agudo de la HTA grave se mencionan en la tabla $8^{7}$. Adicionalmente, la ESC considera que en caso de edema pulmonar secundario a preeclampsia se use nitroglicerina, mientras sugieren que el nitroprusiato de sodio se use como última alternativa, debido al riesgo de toxicidad fetal por cianuro'.

\section{Otros tratamientos}

Se recomiendan esteroides en pacientes con HTA grave que reciben manejo expectante y con edad gestacional menor de 37 semanas $^{3}$. Por otro lado, el sulfato de magnesio no es un antihipertensivo, pero tiene un papel crucial en la prevención de las convulsiones en mujeres con preeclampsia al elevar el umbral convulsivo; así mismo, es la terapia de elección en la paciente con convulsiones por eclampsia. Sin embargo, no hay evidencia contundente para usarlo de rutina en todas las pacientes con preeclampsia sin criterios de 
Tabla 8. Tratamiento antihipertensivo para control urgente en hipertensión arterial grave

\begin{tabular}{|c|c|c|c|}
\hline Medicamento & Dosis & Comentario & Inicio de acción \\
\hline Labetalol & $\begin{array}{l}10-20 \mathrm{mg} \text { IV, seguido de } 20-80 \mathrm{mg} \text { cada } \\
10 \text { - } 30 \text { minutos hasta una dosis } \\
\text { acumulada máxima de } 300 \mathrm{mg} \text {, o infusión } \\
\text { de } 1-2 \mathrm{mg} / \mathrm{min} \text { IV }\end{array}$ & $\begin{array}{l}\text { Evitar en mujeres con asma, enfermedad } \\
\text { miocárdica persistente, función cardiaca } \\
\text { descompensada, bloqueo cardíaco avanzado y } \\
\text { bradicardia. }\end{array}$ & $1-2$ minutos \\
\hline Hidralazina & $\begin{array}{l}5 \mathrm{mg} \text { IV o IM, luego } 5 \text { - } 10 \mathrm{mg} \text { IV cada } 20 \\
-40 \text { minutos hasta una dosis acumulada } \\
\text { máxima de } 20 \mathrm{mg} \text {, o infusión de } \\
0,5-10 \mathrm{mg} / \text { hora }\end{array}$ & $\begin{array}{l}\text { Las dosis más altas o más frecuentes se asocian } \\
\text { con hipotensión materna, cefalea y trazo anormal } \\
\text { en la frecuencia cardiaca fetal. }\end{array}$ & $10-20$ minutos \\
\hline $\begin{array}{l}\text { Nifedipina oral } \\
\text { (liberación } \\
\text { inmediata) }\end{array}$ & $\begin{array}{l}10-20 \mathrm{mg} \text { V0, repetir a las } 20 \text { minutos si } \\
\text { es necesario, luego } 10-20 \mathrm{mg} \text { cada } 2-6 \\
\text { horas, dosis diaria máxima de } 180 \mathrm{mg}\end{array}$ & Puede presentarse taquicardia refleja y cefalea & $5-10$ minutos \\
\hline
\end{tabular}

gravedad. En preeclampsia grave se recomienda recibir sulfato de magnesio intravenoso para profilaxis de eclampsia y continuar la administración intra y postparto ${ }^{3,7,17}$. Se recomienda dosis de carga de 4 gramos intravenosos en 10 a15 minutos, seguido de infusión de $1 \mathrm{gramo} /$ hora durante 24 horas. El uso de magnesio requiere estrecho seguimiento del gasto urinario, frecuencia respiratoria y reflejos tendinosos profundos ${ }^{3}$.

La tabla 9 resume las recomendaciones generales del manejo de los trastornos hipertensivos asociados al embarazo acorde con las guías ESC'1

\section{Eclampsia}

Es una emergencia obstétrica y la prioridad es estabilizar a la madre. La mayoría de las convulsiones eclámpticas son autolimitadas; no obstante, el sulfato de magnesio debe iniciarse tan pronto sea posible según el protocolo enunciado anteriormente ${ }^{3}$. Si la paciente ya venía recibiendo sulfato de magnesio, debe administrarse otro bolo de $2-4$ gramos en 5 minutos. La HTA debe ser controlada vía intravenosa como se discutió previamente?.

Tan pronto la condición de la madre se considere estable, se debe considerar el parto por la vía más conveniente, vaginal en situaciones seleccionadas, si bien la más usada en este escenario es la cesárea. Todas las mujeres con eclampsia deben ser manejadas en centros de referencia ${ }^{3}$.

\section{Síndrome HELLP}

Los síntomas incluyen molestias abdominales, como dolor, sensibilidad en el cuadrante superior derecho o epigastrio, náuseas, vómito e ictericia; también puede presentarse cefalea y cambios visuales ${ }^{2,7}$. Cuando se sospeche síndrome HELLP se recomienda realizar hemograma, extendido de sangre periférica, lactato deshidrogenasa (LDH) y panel metabólico completo. Puede encontrarse hemólisis con eritrocitos anormales, LDH elevada, bilirrubina indirecta elevada como marcador de hemólisis y plaquetas menores de $100.000 /$ microlitro $^{1,7-9}$. Esto constituye una emergencia obstétrica, en la que el manejo expectante se asocia con mayor morbilidad materna, por lo que las pacientes en esta situación deben llevarse a terminación de la gestación $n^{3,7}$.

\section{Momento del parto}

El tratamiento definitivo de los trastornos hipertensivos asociados al embarazo y especialmente de la preeclampsia y sus presentaciones más graves (HELLP y eclampsia) es el alumbramiento de la placen$t^{2,10}$. El momento del parto se define analizando los riesgos de prematuridad para el feto y el riesgo materno de continuar con la gestación ${ }^{7,9}$.

En el caso de enfermedad materna que ponga en riesgo la vida, como HTA grave refractaria al tratamiento, eclampsia, abrupcio placentario, deterioro rápido en el contexto de síndrome HELLP o disfunción renal, el embarazo debe terminarse ${ }^{3,7}$.

Si el embarazo es menor a 34 semanas y el parto puede ser diferido en forma segura, debe realizarse vigilancia hospitalaria estrecha, administración de esteroides para maduración pulmonar fetal y, según la evolución de la presión arterial, aparición de signos de gravedad o compromiso fetal, la terminación de la 
Tabla 9. Recomendaciones de la Sociedad Europea de Cardiología para el tratamiento de la hipertensión arterial en el embarazo

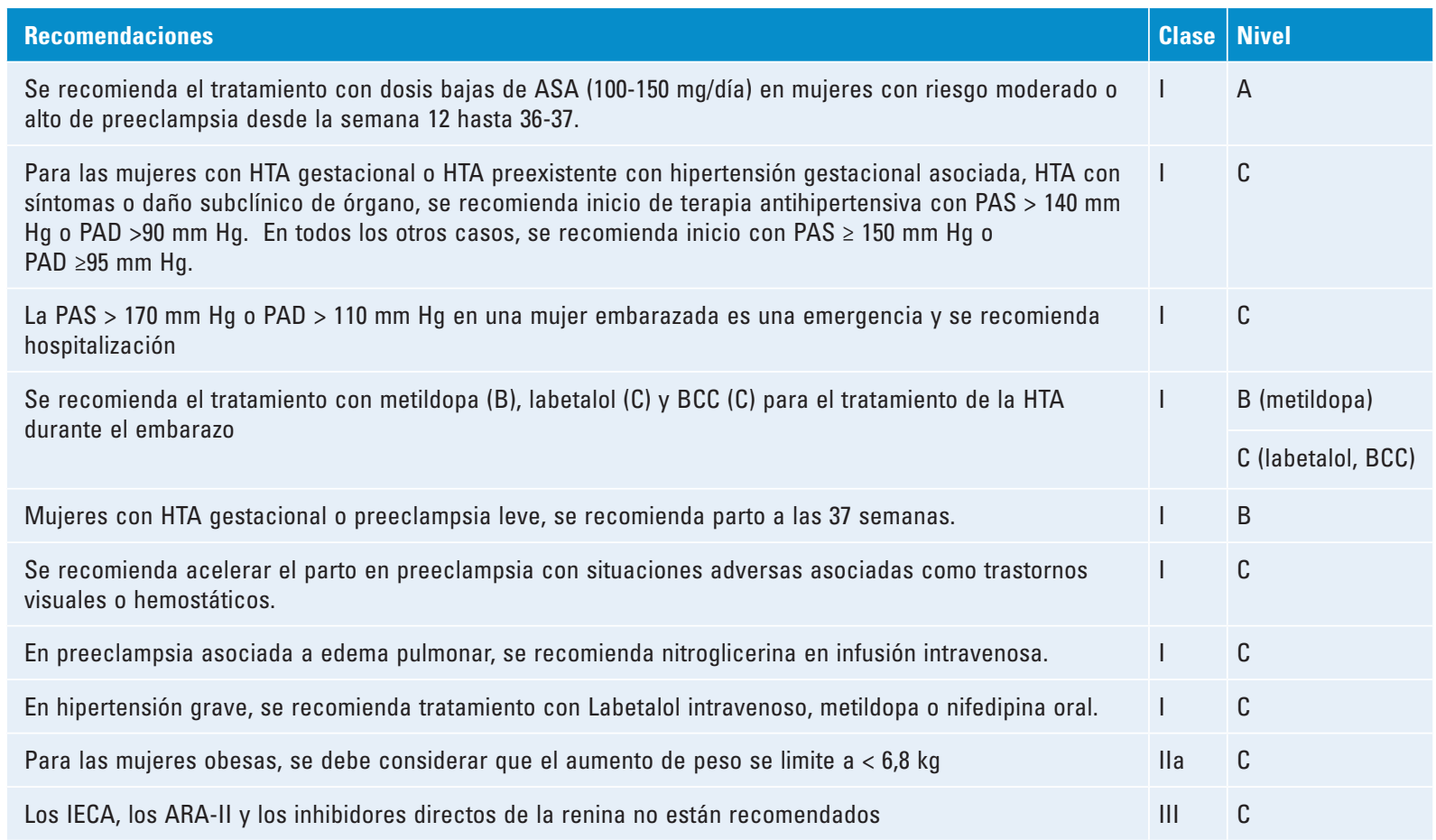

ARA-II: antagonistas del receptor de angiotensina II; ASA: ácido acetilsalicílico; BCC: bloqueadores de canales de calcio; HTA: hipertensión arterial; IECA: inhibidores de la enzima convertidora de angiotensina; PAD: presión arterial diastólica; PAS: presión arterial sistólica.

gestación. Una vez estabilizada la madre, con adecuado equipo de anestesia, obstetricia y neonatal se procede con el parto en forma segura si está indicado $0^{7,17}$.

Entre las 34-37 semanas, se debe mantener vigilancia hospitalaria estrecha, realizar el protocolo de maduración pulmonar y considerar el parto ante cualquier manifestación de gravedad (Tabla 2) $)^{7,17}$. En caso de embarazo a término ( $\geq 37$ semanas) este se terminará en las siguientes 24-48 horas, iniciando sulfato de magnesio de acuerdo con el protocolo mencionado previamente si existen criterios de gravedad ${ }^{3}$.

En las mujeres con HTA crónica con condiciones materno-fetales estables, se sugiere manejo expectante y terminación del embarazo después de la semana 37 , en caso que requiera medicación antihipertensiva, y después de la semana 38 de gestación si está controlada adecuadamente sin terapia antihipertensiva?.

En mujeres que requieren analgesia para el trabajo de parto $o$ anestesia para la cesárea y las condiciones lo permiten, se recomienda anestesia neuroaxial (espinal o epidural). No se recomienda uso rutinario de monitorización invasiva. La ergometrina (empleada en la prevención y el tratamiento de la hemorragia posparto) debe evitarse en mujeres con HTA debido a la posibilidad de aumentar las cifras tensionales, mientras que la oxitocina (también empleada para reducir el sangrado luego del parto) puede utilizarse según los protocolos obstétricos habituales ${ }^{3}$.

\section{Manejo posparto}

En todas las mujeres (no solo en aquellas con trastornos hipertensivos) se recomienda dar instrucciones precisas sobre los síntomas y signos de preeclampsia en el postparto. En embarazos normales, la presión arterial disminuye luego del parto, pero pueden presentarse elevaciones transitorias en el posparto secundario al dolor, medicamentos, exceso de líquidos, movimiento del volumen al compartimiento intravascular o restauración del tono vascular; por lo tanto, se debe continuar control estricto de la $\mathrm{PA}^{21}$. Las pacientes que estuvieron normotensas en el embarazo pueden presentar trastornos hipertensivos asociados al embarazo en el periodo posparto, con una prevalencia que no es clara, ya que hay reportes entre 0,3$27,5 \%$. 
En pacientes con HTA persistente postparto $\geq 150 / 100$, se sugiere iniciar terapia antihipertensiva; se recomienda el manejo intravenoso si la presión arterial es $\geq 160 / 110^{7,22}$. Así mismo, en pacientes que ya tenían un trastorno hipertensivo, se buscarán las metas previamente enunciadas ${ }^{3,7,9,17}$. Si ocurren nuevamente manifestaciones de enfermedad grave, la madre deberá ser trasladada a un centro de referencia y administrar sulfato de magnesio por lo menos 24 horas, para profilaxis de la eclampsia ${ }^{22}$.

Un aspecto por resaltar es que en el periodo posparto se pueden iniciar medicamentos que no eran permitidos en el embarazo, pero que son seguros en la lactancia, como los IECA o los ARA-II, aunque para mayor seguridad se recomienda consultar sitios web especializados en medicamentos en la lactancia, como http://www.e-lactancia.org. La metildopa debe evitarse en el postparto debido a riesgo de depresión y sedación ${ }^{1}$.

Si la presión arterial está bien controlada y no existen manifestaciones de gravedad, la madre puede ser dada de alta, aunque en la primera semana deberá controlarse la presión arterial al menos cada dos días y luego semanalmente. La medicación puede ser reducida y luego suspendida según las cifras tensionales. En forma ambulatoria, se debe continuar medicación antihipertensiva hasta que la presión arterial se normalice (usualmente requiere tratamiento por 3-4 semanas). Luego se recomienda continuar control de la presión arterial cada 1-2 semanas por 1 mes y luego cada 3 a 6 meses por 1 año. La HTA que persiste más de 6 semanas en el postparto representa una enfermedad no asociada directamente con la gestación, y puede tratarse de HTA esencial o la presencia de causas secundarias, por lo que estas pacientes requieren estudio con MAPA y ser referidas para los estudios pertinentes según el caso. La proteinuria que persiste más allá de 6-12 semanas en el posparto requiere estudios adicionales, especialmente en pacientes con preeclampsia de inicio temprano por sospecha de enfermedad renal de base bi,22. $^{21}$.

\section{Riesgo cardiovascular a futuro}

Las mujeres que presentan HTA en su primer embarazo tienen aumento del riesgo en futuros embarazos. Cuanto más temprano el inicio de HTA en el primer embarazo, mayor riesgo de recurrencia en futuros embarazos. El antecedente de preeclampsia está asociado con anomalías metabólicas, vasculares y riesgo futuro para HTA, diabetes mellitus, y enfermedad cardiovascular y renal. Se recomiendan modificaciones del estilo de vida para evitar complicaciones en futuros embarazos y reducir el riesgo cardiovascular, además de una visita anual para el control de la presión arterial y metabólico ${ }^{23-26}$.

\section{Conclusión}

Los trastornos hipertensivos asociados al embarazo son una complicación cardio-obstétrica frecuente, por lo que todo médico que evalúa o potencialmente puede evaluar gestantes, debe estar familiarizado con su diagnóstico y tratamiento, para definir la remisión a centros de referencia en los casos que se requiera.

Es fundamental el seguimiento estricto de las cifras tensionales, síntomas y pruebas de laboratorio para detectar en forma temprana pacientes con riesgo alto, optimizar el cuidado y definir el momento apropiado para el parto.

En el posparto pueden presentarse o empeorar estas enfermedades, por tanto, todas las mujeres hipertensas o no deben recibir instrucciones precisas sobre controles, signos y síntomas de alarma para el periodo posparto.

Los antecedentes ginecoobstétricos hacen parte de la historia cardiovascular de una mujer, dado que la historia de preeclampsia es un marcador de riesgo cardiovascular futuro.

\section{Financiamiento}

Los autores declaran que este artículo no requirió financiación.

\section{Conflicto de intereses}

Los autores declaran que no hay conflicto de intereses.

\section{Responsabilidades éticas}

Protección de personas y animales. Los autores declaran que para esta investigación no se han realizado experimentos en seres humanos ni en animales.

Confidencialidad de los datos. Los autores declaran que en este artículo no aparecen datos de pacientes.

Derecho a la privacidad y consentimiento informado. Los autores declaran que en este artículo no aparecen datos de pacientes.

\section{Bibliografía}

1. Regitz-Zagrosek V, Roos-Hesselink J, Bauersachs J, Blomström-Lundqvist C, Cífková R, De Bonis M et al. 2018 ESC Guidelines for the management of cardiovascular diseases during pregnancy. The Task Force 
for the Management of Cardiovascular Diseases during Pregnancy of the European Society of Cardiology. Eur Heart J. 2018;39: 3165-241.

2. Townsend R, O'Brien $P$, Khalil A. Current best practice in the management Of hypertensive disorders in pregnancy. Integrated Blood Pressure Control. 2016:979-94.

3. Representantes del grupo desarrollador de la guía - Universidad Nacional de Colombia - Alianza CINETS. Guía de la práctica clínica para e abordaje de las complicaciones hipertensivas asociadas al embarazo. Rev Colomb Obstet Ginecol. 2013;64:289-326.

4. Robers J, August P, Bakris G, Barton J, Bernstein I, Druzin M, et al. Hypertension in Pregnancy Report of the American College of Obstetricians and Gynecologists'. Task Force on Hypertension in Pregnancy. Obstet Ginecol. 2013;122:1122-31.

5. Williams B, Mancia G, Spiering W, Rosei EA, Azizi M, Burnier M et al. 2018 ESC/ASH guidelines for the management of arterial hypertension: The task force for the management of arterial hypertension of the European Society of Cardiology (ESC) and the European Society of Hypertension (ESH). Eur Heart J. 2018;39:3021-104.

6. Whelton PK, Carey RM, Aronow WS, Casey DE Jr, Collins KJ, Dennison Himmelfarb C, et al. 2017 ACC/AHA/AAPA/ABC/ACPM /AGS/APhA / ASH/ASPC /NMA/PCNA Guideline for the Prevention, Detection, Evaluation, and Management of High Blood Pressure in Adults: Executive Summary: A Report of the American College of Cardiology/American Heart Association Task Force on Clinical Practice Guidelines. J Am Soc Hypertens. 2018;12:579.e1-e73.

7. ACOG practice bulletin number 202. Gestational hypertension and preeclampsia. Obstet Gynecol. 2019;133:e1-e25.

8. ACOG practice bulletin number 203. Chronic hypertension in pregnancy. Obstet Gynecol. 2019;133:e26-e50.

9. Butalia S, Audibert F, Cote AM, Firoz T, Logan AG, Magee LA et al. Hypertension Canada's 2018 guidelines for the management of Hypertension in pregnancy. Can J Cardiol. 2018;34:526-31.

10. Braunthal S, Brateanu A. Hypertension in pregnancy: pathophysiology and treatment. SAGE Open Med. 2019;7:1-15.

11. Zeisler $H$, Llurba $E$, Chantraine $F$, Vatish $M$, Cathrine $A$, Sennström $M$ et al. Predictive value of the sFIt-1:PIGF ratio in women with suspected preeclampsia. N Engl J Med. 2016;374:13-22.

12. Vaught AJ, Kovell LC, Szymanski LM, Mayer SA, Seifert SM, Vaidya D, et al. Acute cardiac effects of severe pre-eclampsia. J Am Col Cardiol. 2018;72:1-11.

13. O'Brien E, Parati G, Stergiou G, Asmar R, Beilin L, Bilo G, et al. European Society of Hypertension Position Paper on Ambulatory Blood Pressure Monitoring. J Hypertens. 2013;31:1731-68.
14. Gijón-Conde T, Gorostidi M, Benegas JR, de la Sierra A, Segura J, Vinyoles $\mathrm{E}$ et al. Documento de la Sociedad Española de Hipertensión - Liga Española para la lucha contra la hipertensión arterial (SHE LELHA) sobre monitorización ambulatoria de la presión arterial (MAPA) 2019. Hipertens Riesgo Vasc. 2019;36:199-212.

15. Rolnik DL, Wright D, Poon LC, O'Gorman N, Syngelaki A, Matallana C, et al. Aspirin versus placebo in pregnancies at high risk for preterm preeclampsia. N Engl J Med. 2017;377:613-22.

16. Hofmeyr GJ, Lawrie TA, Atallah AN, Duley L, Troloni MR. Calcium supplementation during pregnancy for preventing hypertensive disorders and related problems. Cochrane Database Syst Rev. 2014;6:CD001059.

17. National Institute for Health and Care Excellence. Hypertension in pregnancy: diagnosis and management (NICE guideline NG133). 2019. Disponible en: http://www.nice.org.uk/guidance/ng133.

18. Abalos E, Duley L, Steyn DW. Antihypertensive drug therapy for mild or moderate hypertension during pregnancy. Cochrane Database Syst Rev. 2014;2:CD002252.

19. Magee LA, Von Dadelszen P, Rey E, Ross S, Asztalos E, Murphy KE et al. Less-tight versus tight control of hypertension in pregnancy. N Eng J Med. 2015;372:407-17.

20. Magee LA, Von Dadelszen $P$, Singer J, Lee T, Rey E, Ross S et al. The CHIPS randomized trial (Control of Hypertension in Pregnancy Study). Is severe hypertension just an elevated blood pressure? Hypertension. 2016;68:1153-9.

21. Bramham K, Nelson-Piercy C, Brown MJ, Chappell L. Postpartum management of hypertension. BMJ. 2013;346:e8687.

22. Sibai BM. Etiology and management of postpartum hypertension-preeclampsia. Am J Obstet Gynecol. 2012;206:470-5.

23. Mosca L, Benjamin EJ, Berra K, Bezanson JL, Dolor RJ, Lloyd-Jones DM. Effectiveness-based guidelines for the prevention of cardiovascular disease in women-2011 update: a guideline from the American Heart Association. Circulation. 2011:123:1243-62.

24. Bushnell C, McCullough LD, Awad IA, Chireau MV, Fedder WN Furie KL et al. Guidelines for the prevention of stroke in women: a statement for healthcare professionals from the American Heart Association/ American Stroke Association. Stroke. 2014;45:1545-88.

25. Piepoli MF, Hoes AW, Agewall S, Albus C, Brotons C, Catapano AL, et al. 2016 European guidelines on cardiovascular disease prevention in clinical practice. Eur Heart J. 2016;37:2315-81.

26. Arnett DK, Blumenthal RS, Albert MA, Buroker AB, Goldberger ZD, Hahn EJ et al. 2019 ACC/AHA guideline on the primary prevention of cardiovascular disease. A report of the American College of Cardiology/ American Heart Association Task Force on clinical practice guidelines. Circulation. 2019;140:e596-e646. 